\title{
Labeling of cynomolgus monkey bone marrow-derived mesenchy- mal stem cells for cell tracking by multimodality imaging
}

\author{
REN ZhenHua ${ }^{1,2}$, WANG JiaYin ${ }^{1}$, ZOU ChunLin $^{1}$, GUAN YunQian ${ }^{1} \&$ ZHANG Yu Alex ${ }^{1 *}$ \\ ${ }^{1}$ Cell Therapy Center, Xuanwu Hospital, Capital Medical University, and Key Laboratory of Neurodegeneration, \\ Ministry of Education, Beijing 100053, China; \\ ${ }^{2}$ Department of Anatomy, Anhui Medical University, Hefei 230032, China
}

Received June 28, 2011; accepted September 25, 2011

\begin{abstract}
Recently, transplantation of allogeneic and autologous cells has been used for regenerative medicine. A critical issue is monitoring migration and homing of transplanted cells, as well as engraftment efficiency and functional capability in vivo. Monitoring of superparamagnetic iron oxide (SPIO) particles by magnetic resonance imaging (MRI) has been used in animal models and clinical settings to track labeled cells. A major limitation of MRI is that the signals do not show biological characteristics of transplanted cells in vivo. Bone marrow mesenchymal stem cells (MSCs) have been extensively investigated for their various therapeutic properties, and exhibit the potential to differentiate into cells of diverse lineages. In this study, cynomolgus monkey MSCs (cMSCs) were labeled with Molday ION Rhodamine-B ${ }^{\mathrm{TM}}$ (MIRB), a new SPIO agent, to investigate and characterize the biophysical and MRI properties of labeled cMSCs in vitro and in vivo. The results indicate that MIRB is biocompatible and useful for cMSCs labeling and cell tracking by multimodality imaging. Our method is helpful for detection of transplanted stem cells in vivo, which is required for understanding mechanisms of cell therapy.
\end{abstract}

bone marrow mesenchymal stem cells, MRI, Molday ION Rhodamine-B ${ }^{\mathrm{TM}}$, transplantation, nonhuman primate

Citation: Ren Z H, Wang J Y, Zou C L, et al. Labeling of cynomolgus monkey bone marrow-derived mesenchymal stem cells for cell tracking by multimodality imaging. Sci China Life Sci, 2011, 54: 981-987, doi: 10.1007/s11427-011-4239-x

Recently, stem cell research has enabled the development of numerous stem cell-based therapeutic approaches [1-3]. Mesenchymal stem cells (MSCs) are ethical, practical and biologically appropriate cell populations for cell therapy. In numerous animal and human studies, MSCs show significant potential for tissue regeneration, which requires biosafety and an effective method to detect transplanted cells in vivo [4-7]. Cell tracking in vivo is an important aspect for the development of successful stem cell therapies. Magnetic resonance imaging (MRI) is used to track transplanted stem cells, because of the ability to non-invasively track stem cells for long periods of time [8,9].

To detect transplanted cells by MRI, cells must be la-

*Corresponding author (email: yaz@bjsap.org) beled with a magnetic contrast agent. Currently, various magnetic nanoparticles, such as superparamagnetic iron oxide (SPIO), have been developed for diverse functional analysis in biomedical research [10,11]. An appropriate magnetic nanoparticle must be nontoxic, biocompatible, efficient for intracellular labeling, and highly sensitive to detection such as Feridex, an FDA approved SPIO contrast agent $[12,13]$. However, a current barrier is limited MRI sensitivity, which cannot distinguish regenerative and differentiated cells from exogenous and endogenous transplanted cells. Therefore, development of multimodality imaging is necessary to reveal the in vivo fate of transplanted cells $[14,15]$. By incorporation of an organic fluorescent dye into a silica-coated magnetite core-shell, bifunctional magnetofluorescent nanoparticles have been created and applied 
to MSCs tracking $[16,17]$.

In this study, we investigate the feasibility of labeling cynomolgus monkey MSCs (cMSCs) with Molday ION Rhodamine-B ${ }^{\mathrm{TM}}$ (MIRB), a new SPIO contrast agent, which is visualized by both MRI and fluorescence microscopy, and assessed the potential for imaging and monitoring of MSCs transplantation.

\section{Materials and methods}

\subsection{Cell culture}

Bone marrow aspirates $(5 \mathrm{~mL})$ were collected from the iliac crest of cynomolgus monkeys (Macaca fascicularis). Mononuclear cells (MNCs) were isolated using Ficoll-Paque ${ }^{\mathrm{TM}}$ Plus (StemCell Tech Inc., Vancouver, Canada). MNCs were washed, and plated at $4 \times 10^{5}$ cells $\mathrm{cm}^{-2}$ in Alpha-MEM (Invitrogen, Carlsbad, CA, USA) supplemented with 10\% MSC-qualified fetal bovine serum (GIBCO, USA), $1 \%$ GlutaMAX ${ }^{\mathrm{TM}}-\mathrm{I}$ (Invitrogen, Carlsbad, CA, USA) and 1\% penicillin-streptomycin (Invitrogen, Carlsbad, CA, USA). Medium was replaced every other day until the cells reached $80 \%$ confluence. Adherent cMSCs were harvested using $0.25 \%$ trypsin-EDTA (Invitrogen, Carlsbad, CA, USA) $\left(37^{\circ} \mathrm{C}\right.$ for $\left.3 \mathrm{~min}\right)$, and replated at $1 \times 10^{4}$ cells $\mathrm{cm}^{-2}$ in the medium described above.

\subsection{Cell labeling}

Passage 3 (P3) cMSCs were plated in a 24 well plate $\left(1 \times 10^{4}\right.$ cells $\mathrm{cm}^{-2}$ ) in $0.5 \mathrm{~mL}$ medium and incubated at $37^{\circ} \mathrm{C}$ with $5 \% \mathrm{CO}_{2}$. After the cells adhered overnight, MIRB (BioPAL Inc., Worcester, MA, USA) was added to the medium at the following concentrations: $0,10,20$ and $50 \mu \mathrm{g} \mathrm{Fe} \mathrm{mL}{ }^{-1}$ for $16 \mathrm{~h}$. After incubation, the MIRB-containing medium was removed by aspiration and cMSCs were washed twice with Dulbecco's phosphate-buffered saline (DPBS, Invitrogen, Carlsbad, CA, USA) to remove extracellular MIRB. MIRBlabeled cMSCs were used for subsequent experiments.

\subsection{Intracellular MIRB analysis}

Loading properties of MIRB for cMSCs were evaluated for intracellular MIRB localization and distribution at $20 \mu \mathrm{g}$ $\mathrm{Fe} / \mathrm{mL}$ for $16 \mathrm{~h}$. Cells were DPBS washed twice, fixed with 4\% paraformaldehyde (PFA) and then stained with Prussian Blue to evaluate intracellular Fe distribution by light microscopy. To confirm the intracellular distribution, fluorescent images of MIRB-labeled cMSCs were obtained and compared with those obtained by light microscopy. To calculate labeling efficiency, MIRB-labeled cMSCs were assessed by counting cells positive for Prussian Blue staining, and flow cytometry for rhodamine-labeled cells. The results were expressed as a percentage of MIRB-labeled cMSCs.

\subsection{Flow cytometry analysis}

cMSCs that were labeled with $20 \mu \mathrm{g}$ Fe $\mathrm{mL}^{-1}$ MIRB were collected and washed with cold DPBS twice, and then re-suspended in DPBS containing $1 \%$ bovine serum albu$\min$ (BSA) at $4^{\circ} \mathrm{C}$ and stained with fluorescent antibodies at $4^{\circ} \mathrm{C}$ for $30 \mathrm{~min}$. The presence of MSCs surface markers, CD73 and CD90 as well as the absence of CD34, and CD45 were analyzed using a FACSCalibur flow cytometer (Becton Dickinson Cytometry Systems, San Jose, CA) and Win MDI 2.9 software. All fluorophore- conjugated monoclonal antibodies were purchased from PharMingen (San Diego, CA, USA).

\subsection{Cell proliferation}

Proliferative capacity of MIRB-labeled cMSCs was evaluated by cell counting and a BrdU incorporation assay. Labeled and control cells were seeded at $2 \times 10^{4}$ cells $/ \mathrm{cm}^{2}$, and then harvested at passages 3-6 for counting with a hemocytometer to determine the magnitude of cell expansion. For the BrdU incorporation assay, $1 \times 10^{4}$ cells were seeded into each well of a 24 well plate. After $24 \mathrm{~h}$ incubation, cells were treated with $10 \mu \mathrm{mol} \mathrm{L}{ }^{-1}$ BrdU (Sigma-Aldrich, St. Louis, MO, USA) for another $24 \mathrm{~h}$. Then, cells were fixed and treated with $2 \mathrm{~mol} \mathrm{~L}^{-1} \mathrm{HCl}$ for $40 \mathrm{~min}$, followed by incubation with mouse anti-BrdU (Roche, Inc, Pleasanton, CA, USA) and fluorescent secondary antibodies. The percentage of BrdU-positive cells was determined by counting the positive cells under fluorescence microscopy.

\subsection{Cell differentiation}

cMSCs were differentiated into adipocytes, osteocytes and chondrocytes using differentiation medium (Lonza Walkersville, Inc., Walkersville, MD, USA) following the manufacturer's instructions (http://www.lonza.com). For adipogenic differentiation, cMSCs were plated at $2.1 \times 10^{4}$ cells $\mathrm{cm}^{-2}$ and allowed to reach $100 \%$ confluence, followed by incubation for three cycles in induction/maintenance medium. cMSCs were then cultured for $7 \mathrm{~d}$ in an adipogenic maintenance medium. Cells were fixed with $10 \%$ buffered formalin and stained with Oil red-O. For chondrogenic differentiation, $2.5 \times 10^{5} \mathrm{cMSCs}$ were pelleted at $150 \times g$ and incubated in complete chondrogenic induction medium containing 10 $\mathrm{ng} \mathrm{mL} \mathrm{m}^{-1}$ transforming growth factor- $\beta 3$ for $28 \mathrm{~d}$. Paraffinembedded pellets were sectioned at $5 \mu \mathrm{m}$ and stained with Safranin O. For osteogenic differentiation, cMSCs were plated at $3.1 \times 10^{3}$ cells $\mathrm{cm}^{-2}$ and incubated for $24 \mathrm{~h}$ in proliferation medium. The medium was replaced with an osteogenic induction medium that was exchanged every $3 \mathrm{~d}$ for 21 d. Cells were then fixed and stained with Von Kossa stain to assess mineralization. 


\subsection{Cell transplantation}

Cells were harvested and resuspended at $1.2 \times 10^{5}$ cells $\mu \mathrm{L}^{-1}$ in sterile DPBS and remained on ice for the duration of the transplantation procedure. Three cynomolgus monkeys received MRI-guided stereotaxic intracerebral injections of MIRB-labeled cMSCs, as described elsewhere [18]. All injections were placed into the right side striatum. Each site was injected with $5 \mu \mathrm{L}$ cell suspension, and the total number of transplanted cells was $6 \times 10^{5}$ at each site. The infusion rate was $1 \mu \mathrm{L} \mathrm{min}{ }^{-1}$, and the syringe remained in place for an additional 3 min after injection.

\subsection{MRI}

MRI of anesthetized cynomolgus monkeys was performed 1 week prior to transplantation, and then at 1 and 2 weeks post-transplantation. Animals were pre-medicated with atropine $\left(0.025 \mathrm{mg} \mathrm{kg}^{-1}\right.$, subcutaneously) and anesthetized with ketamine. Animals were then placed in a stereotaxic frame prior to insertion into the MRI scanner. All MRI experiments were performed with a Magnetom Vision $1.5 \mathrm{~T}$ (Siemens, Germany). Typical imaging parameters for in vivo imaging of cerebral anatomy used a multiple spin-echo sequence and T2 weighted sequences, which have been previously used for detection of SPIO-labeled cells $[6,12,19]$, to assess MRI sensitivity of MIRB-labeled cMSCs.

\subsection{Ex vivo intracellular MIRB}

Two weeks after cMSCs transplantation, recipient monkeys were euthanized with pentobarbital $\left(25 \mathrm{mg} \mathrm{kg}^{-1}\right.$, intravenous) and transcardially perfused with heparinized normal saline, followed by $4 \%$ PFA. The brain was removed and immersed in $4 \%$ PFA for $48 \mathrm{~h}$ fixation and then cryoprotected by immersion in a graded $(10 \%-40 \%)$ sucrose/0.1 mol L ${ }^{-1}$ PBS ( $\mathrm{pH} 7.2$ ) solution. Frozen tissue sections (40 $\mu \mathrm{m})$ were prepared using a sliding knife microtome. Sections were stored in a cryoprotectant solution prior to processing. MIRB-labeled cMSCs were assessed by light microscopy for cells positive for Prussian Blue staining and fluorescence microscopy to detect rhodamine-labeled cells.

\section{Results}

\subsection{Intracellular MIRB distribution}

MIRB is an iron oxide-based superparamagnetic contrast reagent with a $50 \mathrm{~nm}$ colloidal size. After incubation with MIRB for $16 \mathrm{~h}$, internalized MIRB was observed in the cytoplasm of cMSCs (Figure 1B), and was stained with Prussian Blue as observed by light microscopy (Figure 1C).

\subsection{Labeling efficiency}

MIRB is labeled with rhodamine $\mathrm{B}$, a fluorescent dye, and can be visualized by fluorescence microscopy. Similarly, the intracellular fluorescent location of MIRB was also in the cytoplasm of cMSCs as observed under a fluorescence microscope, and was observed at the various concentrations of MIRB used for labeling (Figure 2). The percentage of rhodamine B-positive cells was more than $85 \%$ for cells labeled with $10 \mu \mathrm{g} \mathrm{Fe} \mathrm{mL}^{-1} \mathrm{MIRB}$, and up to $95 \%$ for cells labeled with 20 and $50 \mu \mathrm{g} \mathrm{Fe} \mathrm{mL}^{-1}$ MIRB (Figures 3A and B). A similar result was observed with the percentage of positive cells that were stained with Prussian Blue (Figure 3C).

\subsection{Cell surface markers}

To characterize the phenotype of cultured cMSCs after MIRB-labeling, we examined the surface markers CD73 and CD90, which were present on cMSCs as well as an absence of CD34 and CD45 as determined by flow cytometry. The results showed that within three passages after MIRB labeling, no significant difference existed between the phenotypic profile of MIRB-labeled and control cMSCs at a labeling concentration of $20 \mu \mathrm{g} \mathrm{Fe} / \mathrm{mL}$ MIRB (Figure 4).

\subsection{Proliferative capacity}

After MIRB-labeling at a concentration of $20 \mu \mathrm{g} \mathrm{Fe} \mathrm{mL}^{-1}$ MIRB, the proliferative capacity of cMSCs was evaluated by cell counting and a BrdU incorporation assay. Cell expansion decreased by $16.42 \%$ at passage 5 and $18.68 \%$ at passage $6(* P<0.05)$ (Figure $5 \mathrm{~A})$ compared with that of unlabeled cMSCs. A similar result was observed with the BrdU incorporation assay, the percentage of BrdU-positive cells decreased by $7.6 \%$ at passage $4(* P<0.05)$ and $12.43 \%$ at passage $6(* * P<0.01)$ after $24 \mathrm{~h}$ BrdU incorporation (Figure 5B).

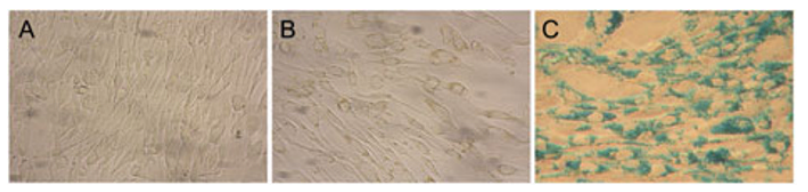

Figure 1 cMSCs labeled with MIRB. Light microscopy images of unlabeled control cMSCs (A), MIRB-labeled cMSCs (B), and Prussian Blue staining of $\mathrm{Fe}(\mathrm{C}), 200 \times$ magnification.

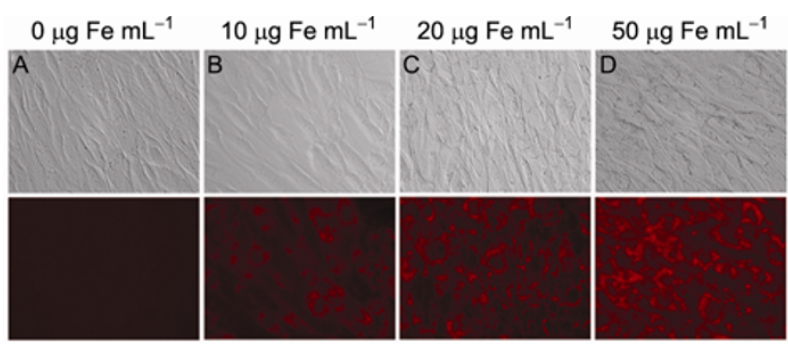

Figure 2 cMSCs labeled with various MIRB concentrations. Fluorescence images of cMSCs cultured with various MIRB concentrations $(0,10$, 20 and $50 \mu \mathrm{g} \mathrm{mL}^{-1}$ ) for $16 \mathrm{~h}, 200 \times$ magnification. 

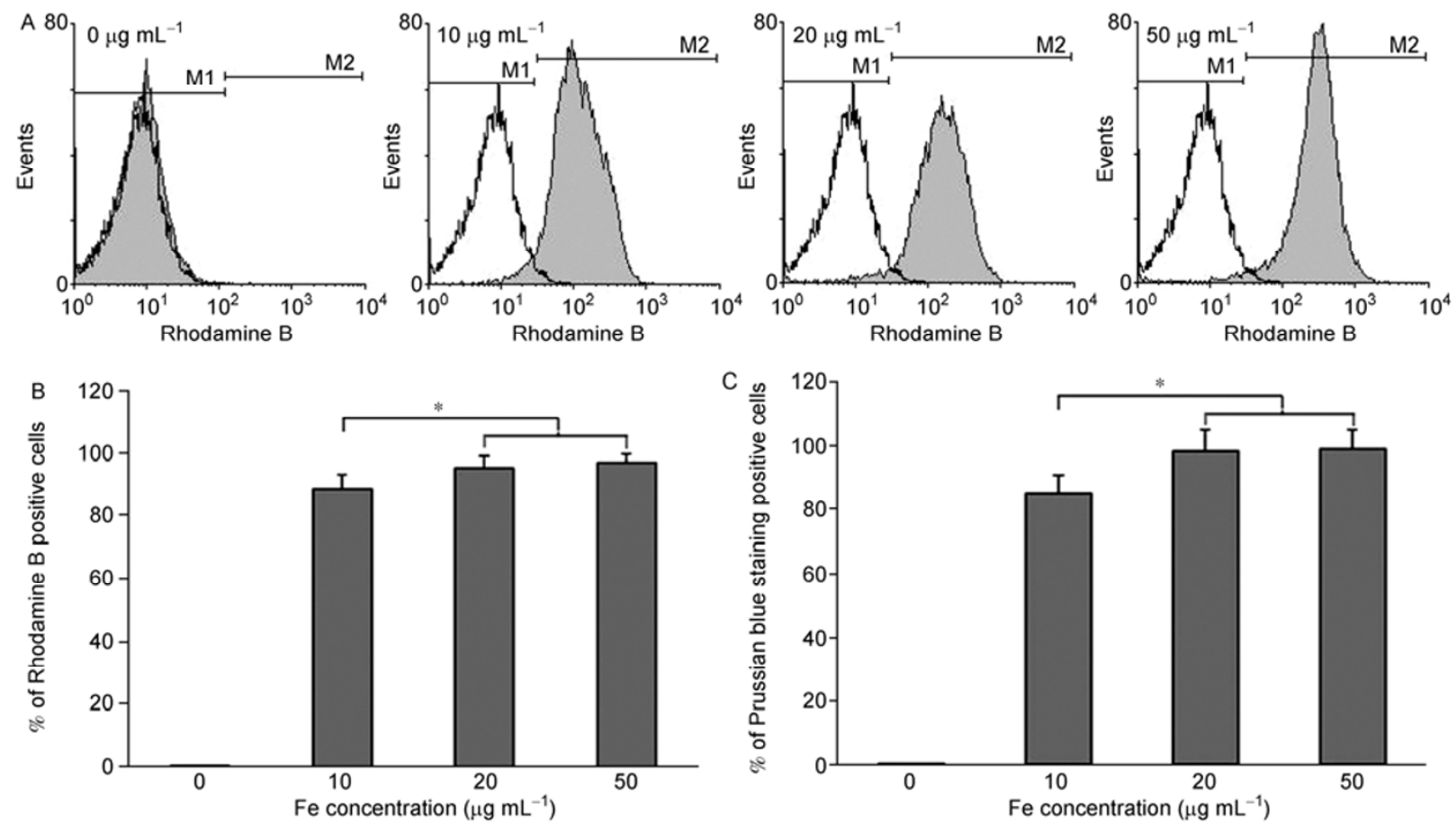

Figure 3 The percentage of positive cells at various MIRB concentrations. The percentage of positive cells was assessed by flow cytometry (A and B) and Prussian Blue staining (C) at various MIRB concentrations $\left(0,10,20\right.$ and $\left.50 \mu \mathrm{g} \mathrm{mL}^{-1}\right)$.
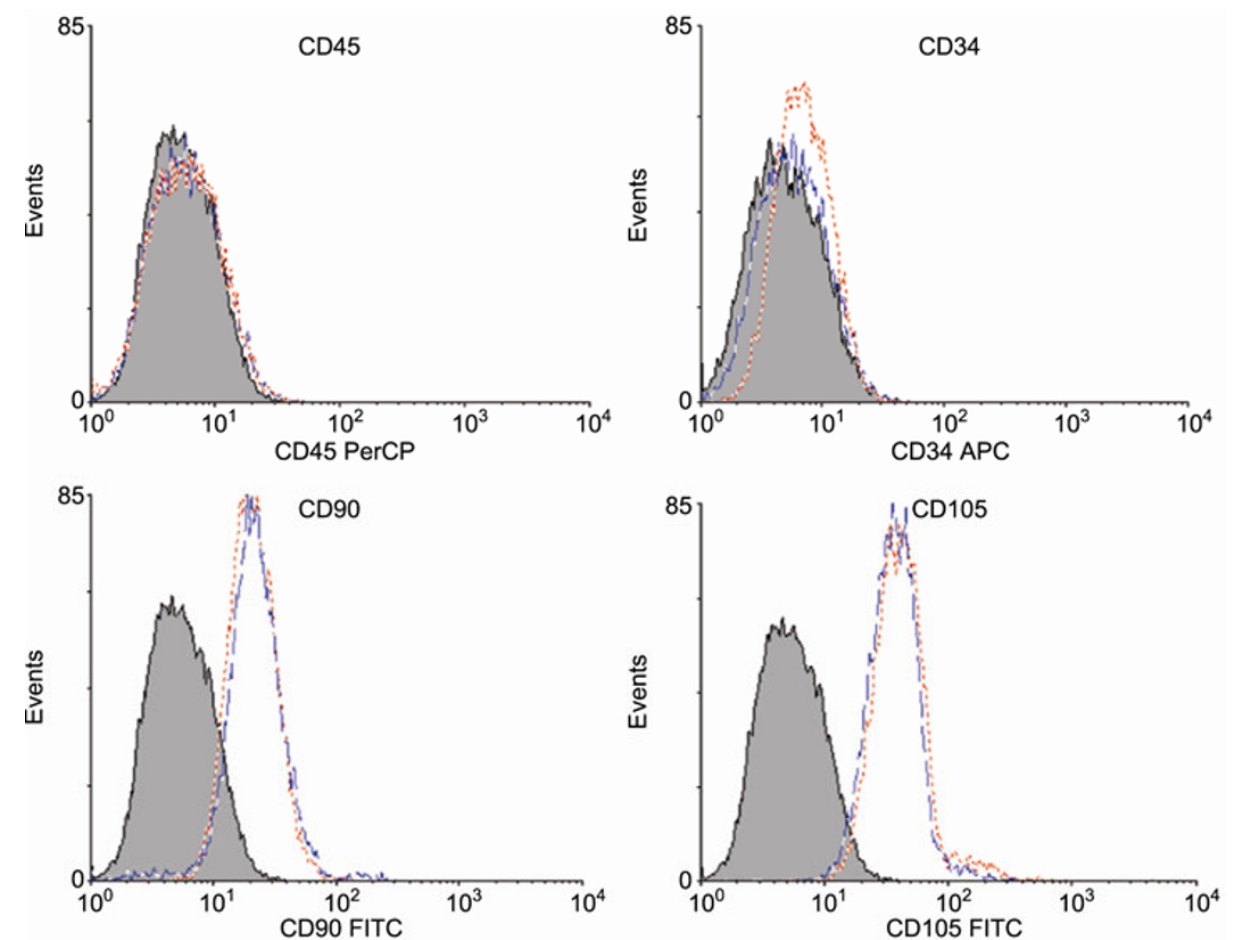

Figure 4 Cell surface markers of MIRB-labeled cMSCs. Cell surface marker expression of CD73 and CD90 is not significantly different between unlabeled (red) and labeled (green) cMSCs as detected by flow cytometry. CD34 and CD45 are not expressed by either group of cMSCs.

\subsection{Differentiation capacity}

To examine the differentiation potential, MIRB-labeled cMSCs (passage 4) were differentiated into osteocytes, ad- ipocytes and chondrocytes. At a labeling concentration of $20 \mu \mathrm{g} \mathrm{Fe} \mathrm{mL} L^{-1} \mathrm{MIRB}$, there was no significant difference between the differentiation potentials of MIRB-labeled and control cMSCs (Figure 6). 

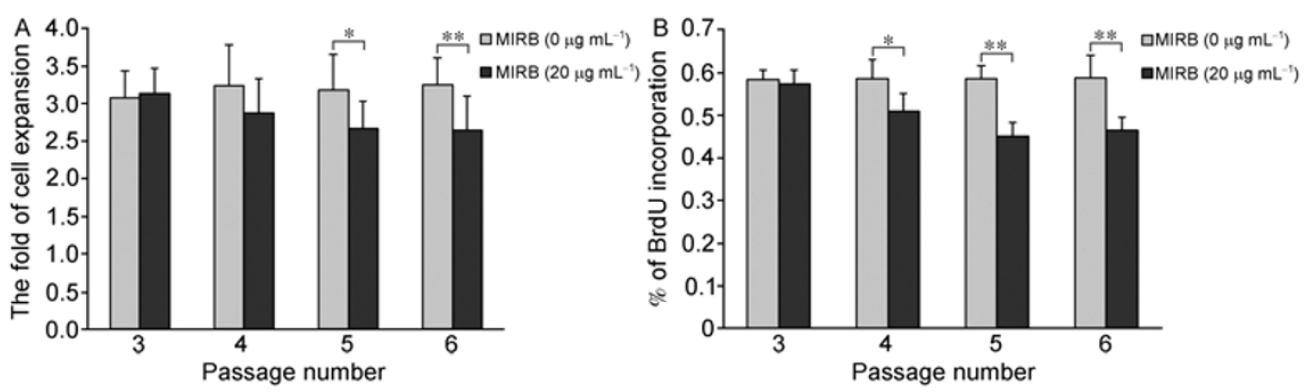

Figure 5 Proliferation capacity of MIRB-labeled cMSCs. Compared with that of unlabeled cMSCs, (A) MIRB-labeled cell proliferation decreased by $16.42 \%$ at passage 5 and $18.68 \%$ at passage $6(* P<0.05)$, and $(\mathrm{B})$ the percentage of BrdU-positive cells in MIRB-labeled cells decreased by $7.6 \%$ at passage $4(* P<0.05)$ and $12.43 \%$ at passage $6(* * P<0.01)$ after $24 \mathrm{~h}$ BrdU incorporation. The percentage of BrdU-positive cells was determined by counting the positive cells in the total number of cells of 10 microscope fields $(100 \times)$.

\subsection{Ex vivo intracellular MIRB}

Following the intracerebral injection of MIRB-labeled and unlabeled cMSCs, MRI based on a multiple spin-echo sequence and T2 weighted sequences was performed with a Magnetom Vision 1.5 T. MRI revealed a significant decrease in signal intensity at the area of MIRB-labeled cell implantation (Figure 7B) compared with that of unlabeled cMSCs (Figure 7A). MRI during the second week after cell

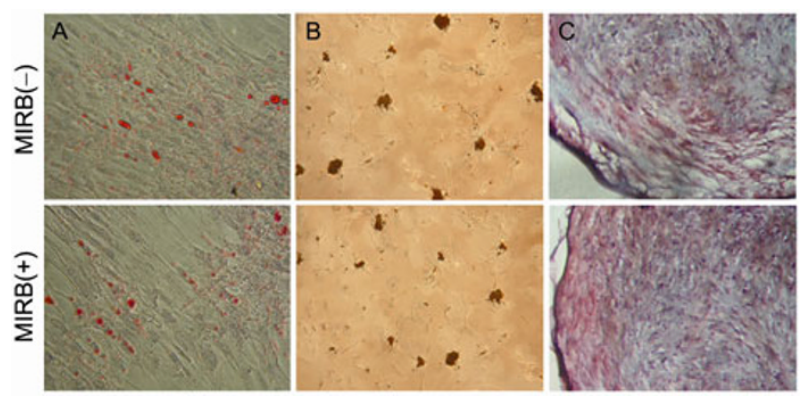

Figure 6 Differentiation capacity of MIRB-labeled cMSCs. Unlabeled (upper row) and MIRB-labeled cMSCs (lower row) (passage 4) were differentiated into adipocytes (A), osteocytes (B) and chondrocytes (C), 100× magnification.
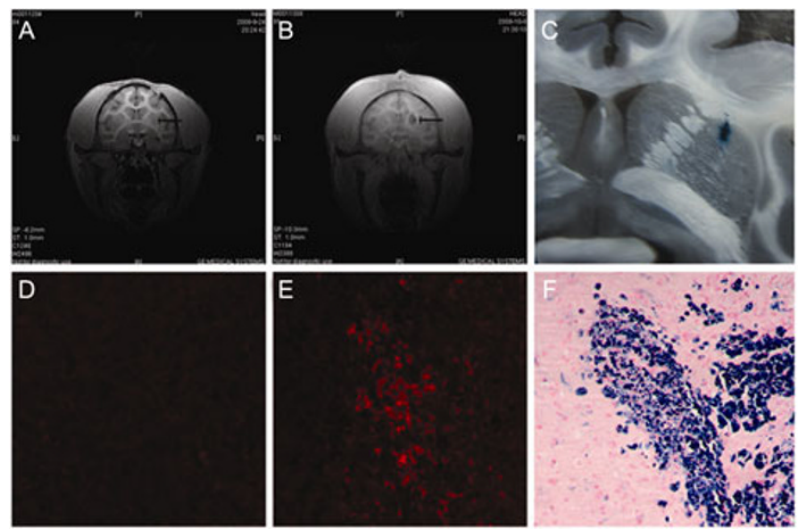

Figure 7 Multimodality imaging of MIRB-labeled cMSCs. Following intracerebral transplantation, MIRB-labeled cMSCs were tracked by MRI (A and B) in vivo, and fluorescence microscopy (D and E) and Prussian Blue staining (C and F) ex vivo. D, E and F, 200× magnification. transplantation showed a low-density area that was slightly reduced, compared with that of the first week (data not shown).

The fluorescence signal of MIRB-labeled cells cannot be detected in vivo. Therefore, monkeys were euthanized with pentobarbital and the brain was removed and cut into $40 \mu \mathrm{m}$ slices. Consistent with MRI results (Figures 7A and B), MIRB-labeled cMSCs were detected in the right striatum of the brain (Figure 7E), which was similar to that of unlabeled cells (Figure 7D). To further detect MIRB-labeled cells, Prussian Blue staining was performed. Similar results were observed with Prussian Blue staining (Figures 7C and F), compared with those of MRI (Figure 7B) and fluorescence microscopy (Figure 7E).

\section{Discussion}

MSCs can be easily isolated and expanded in vitro, and differentiate into various cell types such as osteoblasts, chondrocytes, adipocytes, cardiomyocytes, hepatocytes, endothelial and neuronal cells [20]. The plasticity of MSCs makes them an attractive cell source for stem cell-based therapy. Therefore, in the past decade, MSCs-based therapies are showing significant benefits in multiple clinical trials [21]. However, biological mechanisms of MSCs-mediated cell therapy are unclear in vivo [22]. MSCs may act via paracrine mechanisms, and secrete multiple factors that can regulate endothelial and epithelial permeability, decrease inflammation, enhance tissue repair and inhibit bacterial growth [23,24]. It is generally considered that the field of MSCs-mediated biological mechanisms is significantly less developed than its applications. Therefore, tracking the distribution, migration and differentiation of transplanted MSCs in recipients are important for the development of their therapeutic use.

SPIOs are the most popular contrast agents for tracking and studying stem cells by MRI in various fields such as cancer diagnosis and therapy [25,26], clinical studies and therapy $[27,28]$, and stem cell- based transplantation ther- 
apy [29-31]. However, general SIPOs require the addition of a transfection agent, such as Poly-L-lysine (PLL), for efficient cellular uptake [32]. Moreover, low uptake efficiency of the iron oxide nanoparticles, the cytotoxic effects of SPIO, and the uncertain effect on stem cell proliferation and differentiation, limit their application in clinical and basic research [33-35]. In this study, MIRB demonstrated a high labeling efficiency without a transfection agent, and MIRB-labeling at $20 \mu \mathrm{g} \mathrm{mL}^{-1}$ was less toxic without affecting cell viability, phenotype and differentiation capacity of cMSCs after MIRB-treated cells were incubated in growth medium for various lengths of time. These results indicate that MIRB is biocompatible, and can be used for cell labeling and tracking for cell transplantation.

With technological progress in nanotechnology, magnetic nanoparticles can be nontoxic, biocompatible and efficient for intracellular labeling [36,37] such as Feridex, an FDA approved contrast agent for MRI of adult patients to enhance the T2 weighted images used to detect and evaluate liver lesions [38]. However, limited MRI sensitivity cannot distinguish regenerative and differentiated cells from exogenous and endogenous transplanted cells. Therefore, another imaging modality is required to reveal the biological characteristics of transplanted cells in vivo using confocal laser scanning microscopy [39]. Organic fluorescent dyes have been incorporated into the silica shell for multimodality imaging of transplanted cells. This type of cell tracer has both magnetic and fluorescent properties that enable dual detection of magnetic nanoparticles. Recently, the synthesis of similar nanoparticles for dual modal imaging was reported [40-42]. MIRB is labeled with rhodamine B, a fluorescent dye, and can be visualized by both MRI and fluorescence imaging. The rhodamine $\mathrm{B}$ label excitation wavelength is $555 \mathrm{~nm}$ and the emission wavelength is 565-620 $\mathrm{nm}$, which is commonly used for imaging tissues [43].

Multimodal imaging that enables simultaneous detection by MRI and fluorescence imaging may be a powerful method for in vivo visualization of transplanted stem cells. In this study, we used a multimodality contrast agent, MIRB that was combined with fluorescent molecules to serve as an efficient cell tracking agent for stem cell therapy and research. MIRB efficiently labels cMSCs without significant effects on cell viability, phenotype and differentiation. Importantly, MIRB-labeled cMSCs were detected in vitro and in vivo, and not only traced noninvasively by MRI, but also investigated ex vivo by a fluorescent double-labeling method after animal euthanasia. Our study suggests that MIRB is a useful tracer for cell therapy, and combined with dual fluorescence and MRI features may expand its application in molecular and cellular research.

This work was supported by the National Basic Research Program of China (Grant No. 2007CB947704) and Research Assistance Fund of Anhui Medical University (Grant No. XJ201008).
1 Caplan A I. Review: mesenchymal stem cells: cell-based reconstructive therapy in orthopedics. Tissue Eng, 2005, 11: 1198-1211

2 Dyson S C, Barker R A. Cell-based therapies for Parkinson's disease. Expert Rev Neurother, 2011, 11: 831-844

3 Raikwar S P, Zavazava N. Spontaneous in vivo differentiation of embryonic stem cell-derived pancreatic endoderm-like cells corrects hyperglycemia in diabetic mice. Transplantation, 2011, 91: 11-20

4 Bussolati B, Camussi G. Adult stem cells and renal repair. J Nephrol, 2006, 19: 706-709

5 Daldrup-Link H E, Rudelius M, Piontek G, et al. Migration of iron oxide-labeled human hematopoietic progenitor cells in a mouse model: in vivo monitoring with 1.5-T MR imaging equipment. Radiology, 2005, 234: 197-205

6 Budde M D, Frank J A. Magnetic tagging of therapeutic cells for MRI. J Null Med, 2009, 50: 171-174

7 Adler E D, Bystrup A, Briley-Saebo K C, et al. In vivo detection of embryonic stem cell-derived cardiovascular progenitor cells using Cy3-labeled GadofluorineM in murine myocardium. JACC Cardiovasc Imag, 2009, 2: 1114-1122

8 Guzman R, Uchida N, Bliss TM, et al. Long-term monitoring of transplanted human neural stem cells in developmental and pathological contexts with MRI. Proc Natl Acad Sci USA, 2007, 104: 10211-10216

9 Higuchi T, Anton M, Dumler K, et al. Combined reporter gene PET and iron oxide MRI for monitoring survival and localization of transplanted cells in the rat heart. J Nucl Med, 2009, 50: 1088-1094

10 Chang N K, Jeong Y Y, Park J S, et al. Tracking of neural stem cells in rats with intracerebral hemorrhage by the use of $3 \mathrm{~T}$ MRI. Korean J Radiol, 2008, 9: 196-204

11 Onda T, Honmou O, Harada K, et al. Therapeutic benefits by human mesenchymal stem cells (hMSCs) and Ang-1 gene-modified hMSCs after cerebral ischemia. J Cereb Blood Flow Metab, 2008, 28: 329-340

12 Cai J, Zhang X, Wang X, et al. In vivo MR imaging of magnetically labeled mesenchymal stem cells transplanted into rat liver through hepatic arterial injection. Contrast Media Mol Imag, 2008, 3: 61-66

13 Kim D, Chun B G, Kim Y K, et al. In vivo tracking of human mesenchymal stem cells in experimental stroke. Cell Transplant, 2008, 16: 1007-1012

14 Lu C W, Hung Y, Hsiao J K, et al. Bifunctional magnetic silica nanoparticles for highly efficient human stem cell labeling. Nano Lett, 2007, 7: 149-154

15 Mulder W J, Koole R, Brandwijk R J, et al. Quantum dots with a paramagnetic coating as a bimodal molecular imaging probe. Nano Lett, 2006, 6: 1-6

16 Sung C K, Hong K A, Lin S, et al. Dual-modal nanoprobes for imaging of mesenchymal stem cell transplant by MRI and fluorescence imaging. Korean J Radiol, 2009, 10: 613-622

17 Addicott B, Willman M, Rodriguez J, et al. Mesenchymal stem cell labeling and in vitro MR characterization at $1.5 \mathrm{~T}$ of new SPIO contrast agent: Molday ION Rhodamine- ${ }^{\mathrm{TM}}$. Contrast Media Mol Imaging, 2011, 6: 7-18

18 Emborg M E, Ebert A D, Moirano J, et al. GDNF-secreting human neural progenitor cells increase tyrosine hydroxylase and VMAT2 expression in MPTP-treated cynomolgus monkeys. Cell Transplant, 2008, 17: 383-395

19 Kim S H, Lee W J, Lim H K, et al. SPIO-enhanced MRI findings of well-differentiated hepatocellular carcinomas: correlation with MDCT findings. Korean J Radiol, 2009, 10: 112-120

20 Satija N K, Singh V K, Verma Y K, et al. Mesenchymal stem cell-based therapy: a new paradigm in regenerative medicine. J Cell Mol Med, 2009, 13: 4385-402

21 Wagner J, Kean T, Young R, et al. Optimizing mesenchymal stem cell-based therapeutics. Curr Opin Biotechnol, 2009, 20: 531-536

22 Summer R, Fine A. Mesenchymal progenitor cell research: limitations and recommendations. Proc Am Thorac Soc, 2008, 5: 707-710

23 Lee J W, Fang X, Krasnodembskaya A, et al. Concise review: mesenchymal stem cells for acute lung injury: Role of paracrine soluble factors. Stem Cells, 2011, 29: 913-919

24 Bussolati B, Tetta C, Camussi G. Contribution of stem cells to kidney 
repair. Am J Nephrol, 2008, 28: 813-822

25 Fattahi H, Laurent S, Liu F, et al. Magnetoliposomes as multimodal contrast agents for molecular imaging and cancer nanotheragnostics. Nanomedicine (Lond), 2011, 6: 529-544

26 Fan C, Gao W, Chen Z, et al. Tumor selectivity of stealth multifunctionalized superparamagnetic iron oxide nanoparticles. Int $\mathbf{J}$ Pharm, 2011, 404: 180-190

27 Bulte J W. In vivo MRI cell tracking: clinical studies. AJR Am J Roentgenol, 2009, 193: 314-325

28 Kedziorek D A, Kraitchman D L. Superparamagnetic iron oxide labeling of stem cells for MRI tracking and delivery in cardiovascular disease. Methods Mol Biol, 2010, 660: 171-183

29 Wagner J, Kean T, Young R, et al. Optimizing mesenchymal stem cell-based therapeutics. Curr Opin Biotechnol, 2009, 20: 531-536

30 Karussis D, Karageorgiou C, Vaknin-Dembinsky A, et al. Safety and immunological effects of mesenchymal stem cell transplantation in patients with multiple sclerosis and amyotrophic lateral sclerosis. Arch Neurol, 2010, 67: 1187-1194

31 Addicott B, Willman M, Rodriguez J, et al. Mesenchymal stem cell labeling and in vitro MR characterization at $1.5 \mathrm{~T}$ of new SPIO contrast agent: Molday ION Rhodamine-B ${ }^{\mathrm{TM}}$. Contrast Media Mol Imaging, 2011, 6: 7-18

32 Frank J A, Miller B R, Arbab A S, et al. Clinically applicable labeling of mammalian and stem cells by combining superparamagnetic iron oxides and transfection agents. Radiology, 2003, 228: 480-487

33 Song M, Moon W K, Kim Y, et al. Labeling efficacy of superparamagnetic iron oxide nanoparticles to human neural stem cells: comparison of ferumoxides, monocrystalline iron oxide, cross-linked iron oxide (CLIO)-NH2 and tat-CLIO. Korean J Radiol, 2007, 8: 365-371

34 Hill J M, Dick A J, Raman V K, et al. Serial cardiac magnetic resonance imaging of injected mesenchymal stem cells. Circulation, 2003,
108: 1009-1014

35 Chang J S, Chang K L, Hwang D F, et al. In vitro cytotoxicitiy of silica nanoparticles at high concentrations strongly depends on the metabolic activity type of the cell line. Environ Sci Technol, 2007, 41: 2064-2068

36 Huang H, Xie Q, Kang M, et al. Labeling transplanted mice islet with polyvinylpyrrolidone coated superparamagnetic iron oxide nanoparticles for in vivo detection by magnetic resonance imaging. Nanotechnology, 2009, 20: 365101

37 So $\mathrm{P}$ W, Kalber T, Hunt D, et al. Efficient and rapid labeling of transplanted cell populations with superparamagnetic iron oxide nanoparticles using cell surface chemical biotinylation for in vivo monitoring by MRI. Cell Transplant, 2010, 19: 419-429

38 Mainenti P P, Mancini M, Mainolfi C, et al. Detection of colo-rectal liver metastases: prospective comparison of contrast enhanced US, multidetector CT, PET/CT, and 1.5 Tesla MR with extracellular and reticulo-endothelial cell specific contrast agents. Abdom Imaging, 2010, 35: 511-521

39 Yoon T J, Yu K N, Kim E, et al. Specific targeting, cell sorting, and bioimaging with smart magnetic silica core-shell nanomaterials. Small, 2006, 2: 209-215

40 Lu C W, Hung Y, Hsiao J K, et al. Bifunctional magnetic silica nanoparticles for highly efficient human stem cell labeling. Nano Lett, 2007, 7: 149-154

41 Veiseh O, Sun C, Gunn J, et al. Optical and MRI multifunctional nanoprobe for targeting gliomas. Nano Lett, 2005, 5: 1003-1008

42 Kim J S, Yoon T J, Kim H K, et al. Sentinel lymph node mapping of the stomach using fluorescent magnetic nanoparticles in rabbits. Korean J Gastroenterol, 2008, 51: 19-24

43 Weissleder R. A clearer vision for in vivo imaging. Nat Biotechnol, 2001, 19: 316-317

Open Access This article is distributed under the terms of the Creative Commons Attribution License which permits any use, distribution, and reproduction in any medium, provided the original author(s) and source are credited. 